\title{
Intralabyrinthine Penetrating Ventilation Tube with Preservation of Hearing: An Unusual Clinical Situation
}

\author{
Tantely Razafimahefa Raoelina ${ }^{1}$ Maya Elziere ${ }^{1}$ Justin Michel ${ }^{2}$ Arnaud Devèze $\mathrm{A}^{1,3}$
}

${ }^{1}$ Department of Otolaryngology and Skull Base Surgery, University Hospital Nord, Marseille, France

2 Department of Otolaryngology and Skull Base Surgery, La Timone University Hospital, Marseille, France

${ }^{3}$ Laboratory of Applied Biomechanics IFSTTAR, Aix Marseille

University, Bd Pierre Dramard, Marseille, France
Address for correspondence Arnaud Devèze, MD, PhD, Department of Otolaryngology and Skull Base Surgery, University Hospital Nord, Chemin des bourrely, Marseille 13015, France (e-mail: arnaud.deveze@ifsttar.fr; dr.deveze@gmail.com).

Int Arch Otorhinolaryngol 2015;19:183-186.

\begin{abstract}
Keywords

- labyrinth

- trauma

- tympanic membrane rupture

- perilymph

- stapes surgery

Introduction Traumatic perilymphatic fistula is not a rare event with regards to sport activities or traffic accident. However, iatrogenic damage to the inner ear can occur following the common use of grommets and ventilation tube insertion.

Objectives To report an unusual case of insertion of aeration tube into the vestibule trough the stapes footplate.

Resumed Report A 62-year-old woman experienced iatrogenic penetration into the vestibule from a ventilation tube inserted for retraction pocket management. The event was misdiagnosed both by the surgeon and by the emergency room physicians, leading to delay in the management. However, preservation of the hearing function lasted for 2 weeks prior to deafness, thanks to the valve of Bast, which preserved the cochlear fluid.

Conclusion This case gives us the opportunity to stress the need for systematic clinical examination of traumatic injury to the ear and to recommend performing multiplanar millimetric computed tomography scan with accurate interpretation. Traumatic injuries should be referred to a dedicated traumatic emergency referral center.
\end{abstract}

\section{Introduction}

Traumatic perilymphatic fistula is not a rare event with regards to sport activities or traffic accident. However, iatrogenic damage to the inner ear can occur following the common use of grommets and ventilation tube insertion. Here, we describe how early surgical management may help recover the inner ear function, despite severe damage to this fragile structure.

\section{Review of Literature with Differential Diagnosis}

A perilymph fistula is an abnormal communication between inner ear perilymphatic spaces to the aerated middle ear. A wide variety of signs and symptoms as well as numerous etiologies are associated with perilymph fistula, from congenital malformations to accidental or iatrogenic trauma to the inner ear. ${ }^{1-3}$ During the past 30 years, several cases of traumatic perilymphatic fistula have been reported. Those include cases associated with_ear cleansing, surgeries, trauma, and blast. $^{2-11}$ Traumatic perilymph fistula following penetrating intravestibular ventilation tube has yet to be reported.

\section{Case Report}

A 62-year-old woman with a past history of chronic otitis underwent a tube placement for a right-sided posterior received

May 5, 2014

accepted

June 7, 2014

published online

December 12, 2014
DOI http://dx.doi.org/

10.1055/s-0034-1386505. ISSN 1809-9777.
Copyright $\odot 2015$ by Thieme Publicações License terms Ltda, Rio de Janeiro, Brazil 

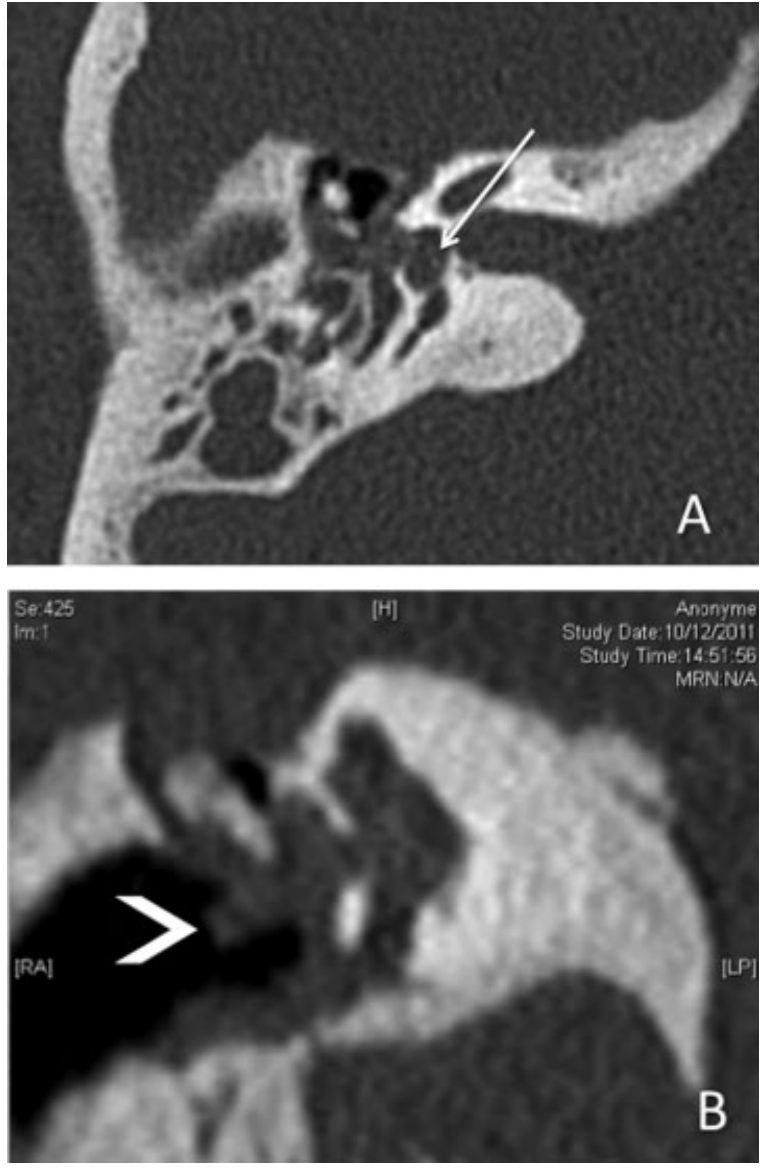

Fig. 1 High-resolution temporal computed tomography (with 0.6-mm slice thickness) in axial (A) and coronal reconstruction (B). The middle ear is filled with fluid; the ossicular chain is not recognizable. There is no pneumolabyrinth, but careful examination may have revealed highdensity components within the vestibule ( $A$, arrow). The tube is seen in the external canal (B, arrowhead).

retraction pocket. Immediately after the procedure, she developed severe vertigo associated with nausea and vomiting. She was discharged with antivomiting medication, which was said to be a normal postoperative event. Four days later she was admitted in emergency for acute vertigo. Clinical evaluation revealed right index finger deviation and left beating nystagmus with hearing loss. Otoscopy showed the tube in place. A temporal bone computed tomography (CT) scan was performed and showed the transtympanic ventilation tube associated with middle ear effusion, but bony components within the vestibule were missed (-Fig. 1). She was admitted in the otolaryngology department and managed medically. Her condition improved, and she was discharged 3 days later.

One week later, she was referred to our tertiary referral center complaining of progressive right-sided hearing loss associated with severe vertigo. She presented with rightsided vestibular impairment and positive Hennebert sign on the right ear. On otoscopic examination, she had tympanic membrane retraction with the ventilation tube inserted in the posterosuperior quadrant with pulsating waterlike fluid leaking through the tube. The audiometry showed a right-sided, severe mixed hearing loss (-Fig. 2). A new

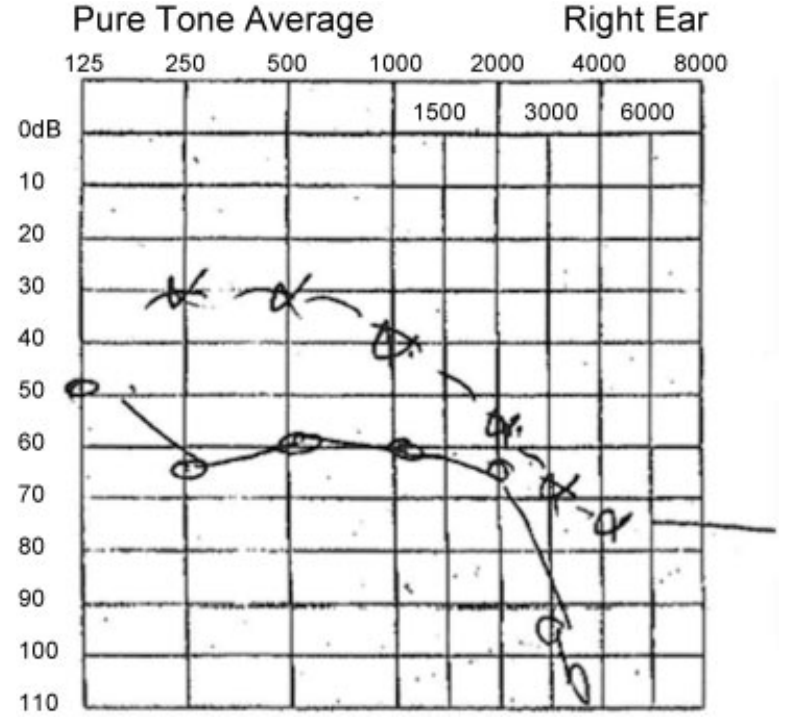

Fig. 2 Right-ear pure tone audiogram performed 1 week after discharge from postemergency hospitalization showing severe mixed hearing loss. Dotted line: bone conduction; plain line: air conduction.
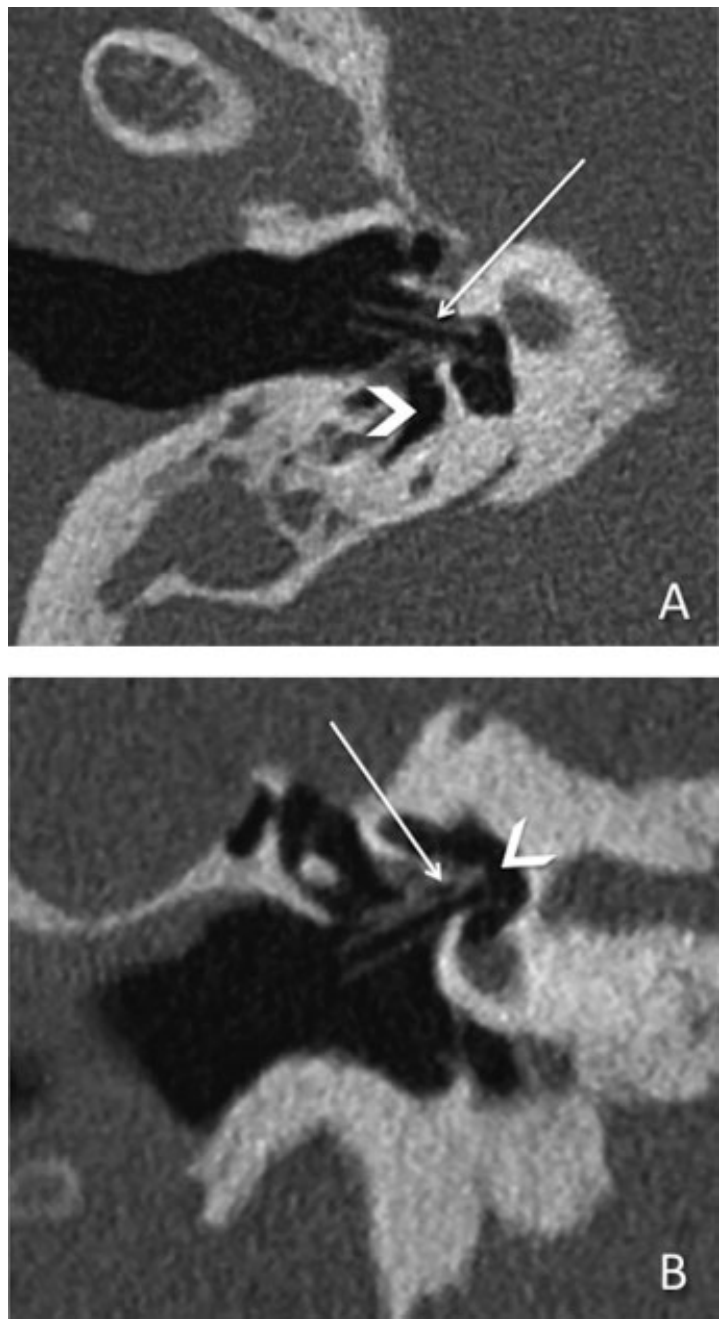

Fig. 3 Second computed tomography scan. The presence of air within the inner ear space (arrowhead) permits the visualization of the tube that penetrates into the vestibule (arrow). 


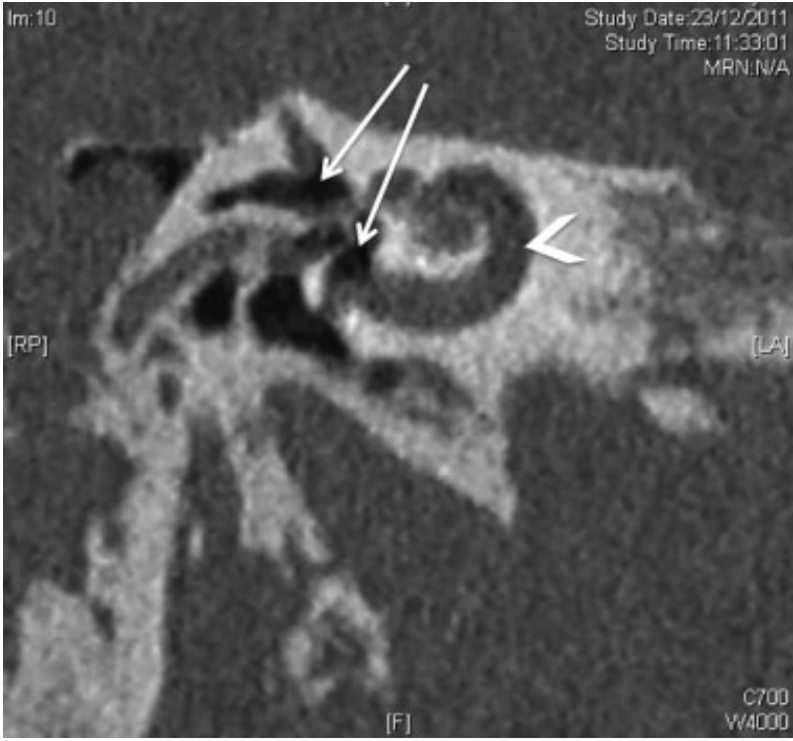

Fig. 4 Second computed tomography scan, coronal view. The pneumolabyrinth extended into the vestibule and the superior and lateral canals (arrows) but did not extend into the cochlea (arrowhead) and might have explained both the conservation of the auditory function up to the 2 weeks after injury as well as the initial recuperation of hearing function after the surgery.

high-resolution temporal bone CT scan clearly showed a right pneumolabyrinth within the vestibule and the semicircular canals, sparing the cochlea, associated with the ventilation tube penetrating the vestibule through the oval window (-Figs. 3 and 4). She was diagnosed with right iatrogenic stapes trauma with perilymph fistula and urgently taken to the operating room. The long process of the incus as well as the stapes posterior crura and the posterior and inferior part of the footplate were fractured. The rest of the footplate was intact. The tube was penetrating the vestibule in the posterior and superior part of the stapes footplate. The tube was gently removed, and the vestibule was refilled with saline solution. The vestibule was sealed with perichondrium and maintained with fibrin glue; the whole montage was stabilized using a Silastic (Dow Corning, USA) sheet positioned from the facial canal to the promontory. The tympanic membrane was repaired using underlay tympanoplasty with cartilage. The ossicular chain was not reconstructed.

In the immediate postoperative period, the patient still presented positional dizziness as well as a left-sided beating nystagmus. Bone conduction auditory testing at postoperative day 2 showed marked improvement of the right-side thresholds (-Fig. 5). The patient was discharged 5 days after surgery. On day 12 after surgery, she suddenly complained of worsening hearing loss and severe tinnitus. The pure tone average (PTA) showed complete anacusis on the right ear (-Fig. 5). No revision surgery was indicated. Treatment with vestibular rehabilitation was introduced and helped dispel the dizziness within a 6 -month period of time.

\section{Discussion}

Our case is singular for the three following points: the erroneous placement of a tube under general anesthesia; the missed diagnosis in the emergency setting; and the relative conservation of the cochlear function despite severe trauma to the labyrinth. This is, to our knowledge, the first direct penetration of an aeration tube into the vestibule. We found another case of labyrinthine injury following tube insertion, due to gradual bony erosion of the osseous labyrinth as a long-term complication. ${ }^{12}$
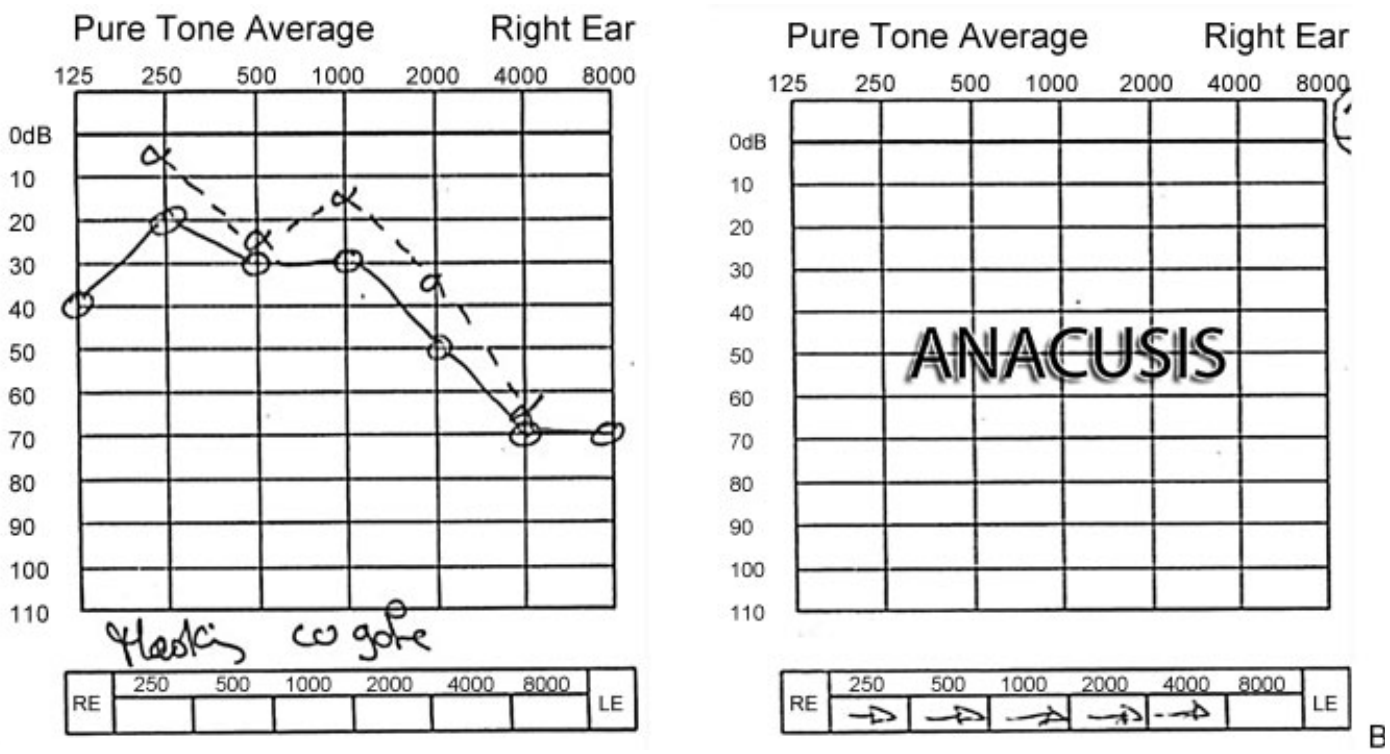

Fig. 5 Right-ear pure tone audiogram done 4 days (A) and 12 days (B) after surgical intervention. (A) Note the hearing improvement in bone conduction. (B) Complete anacusis occurred 12 days after the surgery. Dotted line: bone conduction; plain line: air conduction. Abbreviations: RE, right ear; LE, left ear. 
First, the trauma undoubtedly followed immediate misplacement of the tube, in the posterosuperior quadrant of the drum. This is clearly a wrong procedure, demonstrating an insufficient and inappropriate education. Second, the workup performed in the emergency room was inadequate. Both the clinical history and the CT scan clearly should have stressed the suspicion of trauma to the inner ear. The presence of a pneumolabyrinth on a high-resolution CT scan of the temporal bone is pathognomonic of perilymphatic fistula. ${ }^{8,10-12}$ This inappropriate diagnosis highlights the necessity of appropriate education in trauma-related injuries. Education, reconfiguration of trauma services, and better provision of neurocritical care facilities do improve the quality of care to trauma-related patients. ${ }^{13,14}$ Although the initial CT scan was difficult to interpret, the high-density spots within the vestibule should have drawn attention to a possible severe inner ear trauma (-Fig. 1A). High-resolution CT scan of temporal bone is mandatory in such a scenario. ${ }^{5,6,15-17}$ Finally, it was unexpected that the pneumolabyrinth did not extend into the cochlea (-Fig. 4), but we think that the preservation of the cochlear fluid explains the conservation of the sensorineural component of the hearing. The protective role of the valve of Bast (that closes the utricular duct) explains this unusual situation: decreasing pressure in the whole endolymphatic system collapses the ductus reuniens and causes closure of the valve. ${ }^{18-21}$ It is plausible that the reopening of the valve of Bast after the repair of the fistula may have let the remaining air bubbles $t$ diffuse within the cochlea and induce total auditory loss.

\section{Final Comments}

Tympanic tubes should be inserted in all cases in the infero- or anteroinferior quadrant of the drum, and this procedure should be performed under local anesthesia. In cases of suspicion of traumatic damage to the stapes footplate, surgery should be performed as fast as possible following trauma. Although the outcome of vestibular symptoms is good, regardless of lesion, severity, or intervention, ${ }^{3,6}$ hearing outcome is more unpredictable. We agree with Hidaka et al on the following predictive factors of hearing preservation in a scenario of pneumolabyrinth: (1) early intervention within 15 days; (2) pneumolabyrinth limited to semicircular canals and vestibule; (3) existence of stapes lesion. ${ }^{3}$ According to Tsubota et al, the value of the bone conduction thresholds should be considered a significant prognostic factor as well. ${ }^{6}$

\section{References}

1 Calmels MN, Deguine O. Perilymphatic fistula. Ear Nose Throat J 2004;83(10):666

2 Al Felasi M, Pierre G, Mondain M, Uziel A, Venail F. Perilymphatic fistula of the round window. Eur Ann Otorhinolaryngol Head Neck Dis 2011;128(3):139-141
3 Hidaka H, Miyazaki M, Kawase T, Kobayashi T. Traumatic pneumolabyrinth: air location and hearing outcome. Otol Neurotol 2012; 33(2):123-131

4 Silverstein H, Fabian RL, Stoll SE, Hong SW. Penetrating wounds of the tympanic membrane and ossicular chain. Trans Am Acad Ophthalmol Otolaryngol 1973;77(3):ORL125-ORL135

5 Kojima H, Tanaka Y, Mori E, Uchimizu H, Moriyama H. Penetrating vestibular injury due to a twig entering via the external auditory meatus. Am J Otolaryngol 2006;27(6):418-421

6 Tsubota M, Shojaku H, Watanabe Y. Prognosis of inner ear function in pneumolabyrinth: case report and literature review. Am J Otolaryngol 2009;30(6):423-426

7 Hatano A, Rikitake M, Komori M, Irie T, Moriyama H. Traumatic perilymphatic fistula with the luxation of the stapes into the vestibule. Auris Nasus Larynx 2009;36(4):474-478

8 Rother T, Albrecht C, Issing PR. Pneumolabyrinth after cochlear implantation in large vestibular aqueduct syndrome: a case report. Am J Otolaryngol 2011;32(5):430-432

9 Kaffel N, Jlassi N, Selmi Z, et al. [Traumatic perilymphatic fistulae : about 13 cases]. Tunis Med 2011;89(5):471-475

10 Mandalà M, Colletti L, Carner M, et al. Pneumolabyrinth and positional vertigo after stapedectomy. Auris Nasus Larynx 2011; 38(4):547-550

11 Prisman E, Ramsden JD, Blaser S, Papsin B. Traumatic perilymphatic fistula with pneumolabyrinth: diagnosis and management. Laryngoscope 2011;121(4):856-859

12 Hajiioannou JK, Bathala S, Marnane CN. Case of perilymphatic fistula caused by medially displaced tympanostomy tube. J Laryngol Otol 2009;123(8):928-930

13 Hashmi ZG, Haider AH, Zafar SN, et al. Hospital-based trauma quality improvement initiatives: first step toward improving trauma outcomes in the developing world. J Trauma Acute Care Surg 2013;75(1):60-68, discussion 68

14 Smith NC, Findlay GP, Weyman D, Freeth H. The management of trauma victims with head injury: a study by the National Confidential Enquiry into Patient Outcome and Death. Ann R Coll Surg Engl 2013;95(2):101-106

15 Adil EA, Choudhary AK, Moser KW, Ghossaini SN. Vestibular pneumolabyrinth: why assessment with temporal bone computed tomography utilizing dynamic focal spot mode is important for the diagnosis. Emerg Radiol 2011;18(1):43-45

16 Mafee MF, Valvassori GE, Kumar A, Yannias DA, Marcus RE. Pneumolabyrinth: a new radiologic sign for fracture of the stapes footplate. Am J Otol 1984;5(5):374-375

17 Achache M, Sanjuan Puchol M, Santini L, et al. Late pneumolabyrinth after undiagnosed post-traumatic perilymphatic fistula. Case report illustrating the importance of systematic emergency management. Eur Ann Otorhinolaryngol Head Neck Dis 2013; 130(5):283-287

18 Bast TH. The utriculo-endolymphatic valve. Anat Rec 1928; 40:61-65

19 Bast TH. Function of the utriculo-endolymphatic valve. Two cases of ruptured saccules in children. Arch Otorhinolaryngol 1934; 19:537-550

20 Bachor E, Karmody CS. The utriculo-endolymphatic valve in pediatric temporal bones. Eur Arch Otorhinolaryngol 1995; 252(3):167-171

21 Hofman R, Segenhout JM, Buytaert JA, Dirckx JJ, Wit HP. Morphology and function of Bast's valve: additional insight in its functioning using 3D-reconstruction. Eur Arch Otorhinolaryngol 2008; 265(2):153-157 